\title{
Internal thermal noise in the LIGO test masses: A direct approach
}

\author{
Yu. Levin \\ Theoretical Astrophysics, California Institute of Technology, Pasadena, California 91125
}

(Received 21 July 1997; published 22 December 1997)

\begin{abstract}
The internal thermal noise in LIGO's test masses is analyzed by a new technique, a direct application of the fluctuation-dissipation theorem to LIGO's readout observable, $x(t)=$ (longitudinal position of test-mass face, weighted by laser beam's Gaussian profile). Previous analyses, which relied on a normal-mode decomposition of the test-mass motion, were valid only if the dissipation is uniformally distributed over the test-mass interior, and they converged reliably to a final answer only when the beam size was a non-negligible fraction of the test-mass cross section. This paper's direct analysis, by contrast, can handle inhomogeneous dissipation and arbitrary beam sizes. In the domain of validity of the previous analysis, the two methods give the same answer for $S_{x}(f)$, the spectral density of thermal noise, to within expected accuracy. The new analysis predicts that thermal noise due to dissipation concentrated in the test mass's front face (e.g., due to mirror coating) scales as $1 / r_{0}^{2}$, by contrast with homogeneous dissipation, which scales as $1 / r_{0}\left(r_{0}\right.$ is the beam radius); so surface dissipation could become significant for small beam sizes. [S0556-2821(97)05524-0]

PACS number(s): 04.80.Nn, 05.40. $+\mathrm{j}$
\end{abstract}

\section{INTRODUCTION}

Random thermal fluctuations are expected to be the dominant noise source for the first interferometers in the Laser Interferometer Gravitational Wave Observatory (LIGO) at frequencies between 35 and $100 \mathrm{~Hz}$ [1]. This thermal noise is generally decomposed into a suspension thermal noise and an internal thermal noise for the test masses. The former can be traced back to the friction in the test mass pendular suspension system; the latter is due to internal damping inside the test masses themselves. Traditionally, thermal noise calculations have been based on a normal-mode expansion $[2,3]$. However, Gonzalez and Saulson have also performed an exact calculation of the suspension thermal noise by applying directly the fluctuation-dissipation (FD) theorem [4] in its most general form, due to Callan and Welton [5]. The purpose of this paper is to use the general method of Gonzalez and Saulson to calculate the internal thermal noise (also, [6] has a somewhat complementary to this paper treatment of the internal thermal noise).

In Sec. II we analyze a general situation when a measuring device (e.g., a laser interferometer) monitors the displacement of the surface of a test mass whose internal degrees of freedom are in thermal equilibrium with each other. We develop a general formalism for using the FD theorem to calculate the thermal noise in the most general surface readout quantity. In brief our method is as follows.

To work out the thermal noise at a particular frequency $f$, one should mentally apply pressure oscillating at this frequency to the observed surface of the test mass. The spatial variation of this pressure should mimic that of the light beam intensity (for example, in the case of a Gaussian beam this oscillating pressure has a Gaussian profile of the same width as the beam). The thermal noise is then given by

$$
S_{x}(f)=\frac{2 k_{B} T}{\pi^{2} f^{2}} \frac{W_{\mathrm{diss}}}{F_{0}^{2}},
$$

where $k_{B}$ and $T$ are Boltzmann's constant and the tempera- ture of the mirror, respectively, $F_{0}$ is the amplitude of the oscillating force applied to the surface (i.e., the pressure integrated over the surface), and $W_{\text {diss }}$ is the time-averaged power dissipated in the test mass when this oscillating pressure is applied.

To demonstrate the computational power of this general approach, in Sec. III we consider the case of a cylindrical fused silica test mass monitored by a circular Gaussian laser beam. For the case when the radius of the beam is much less than the size of the the test mass and the dissipation is uniformly distributed throughout the test-mass volume, we derive an analytical expression for the thermal noise [cf. Eq. (15) of Sec. III ]:

$$
S_{x}(f)=\frac{4 k_{B} T}{f} \frac{1-\sigma^{2}}{\pi^{3} E_{0} r_{0}} I \phi\left[1+O\left(\frac{r_{0}}{R}\right)\right] .
$$

Here $\sigma, E_{0}$, and $\phi$ are the Poisson ratio, Young's modulus, and dissipational loss angle [Eq. (11)] of the test-mass material, $r_{0}$ is the radius of the laser beam (which is defined here as a radius at which the intensity of light is $1 / e$ of the maximum intensity), $R$ is a characteristic size of the test mass, and $I=1.87322 \ldots$ in the case of a Gaussian beam. Putting numbers in Eqs. (1) and (2), we find that our results are in agreement with those of Raab and Gillespie [3], who used the more complicated and computationally involved method of normal-mode decomposition. It is interesting to note that as $r_{0} / R$ tends to zero, our simple analytical formula becomes more precise, whereas the more complicated and computationally involved method of normal-mode decomposition requires summing over a larger number of modes and thus becomes computationally more expensive.

Not only can the normal-mode decomposition be computationally expensive, it can also be misleading. We demonstrate this point in Sec. IV by considering a test mass which has a lossy surface, e.g., due to a lossy mirror coating. We estimate the contribution of the surface to the thermal noise using the general method of Sec. II, and show that it differs from the estimate obtained by the method of normal modes 
(which gives a result too small by a factor of at least $\left.\sim r_{0} / R\right)$. This breakdown of the normal-mode analysis will in general happen when the sources of friction are not distributed homogeneously over the test mass. The fundamental reason is that in this case different normal modes can have a common Langevin driving force (which is not so if the defects are distributed homogeneously).

Our analysis shows that thermal noise due to surface losses near the laser beam spot scales as $S_{x}(f) \propto 1 / r_{0}^{2}$, whereas thermal noise due to volume losses scales as $1 / r_{0}$. Correspondingly, for small beam spots the surface losses could become significant. To protect against this, it is important to keep the surface near the laser beam spot as free of potential sources of friction as possible.

\section{GENERAL METHOD}

For concreteness, consider a situation where LIGO's laser beam is shining on the circular surface of one of LIGO's cylindrical test masses. The phase shift of the reflected light contains information about the motion of the test-mass surface. The variable read out by this procedure can be written as

$$
x(t)=\int f(\vec{r}) y(\vec{r}, t) d^{2} r
$$

Here $\vec{r}$ is the transverse location of a point on the test-mass surface, and $y(\vec{r}, t)$ is the displacement of the boundary along the direction of the laser beam at point $\vec{r}$ and time $t$. The form factor $f(\vec{r})$ depends on the laser beam profile and is proportional to the laser light intensity at the point $\vec{r}$ [3]; it is normalized by $\int f(\vec{r}) d^{2} r=1$.

The internal thermal noise of the test mass is defined as the fluctuations in $x(t)$, and our objective is to find the spectral density $S_{x}(f)$ of these fluctuations. We assume that the test mass is in thermal equilibrium at temperature $T$.

Callen and Welton's generalized fluctuation-dissipation theorem [5] says that the spectral density of the fluctuations of LIGO's readout variable $x(t)$ is given by the formula

$$
S_{x}(f)=\frac{k_{B} T}{\pi^{2} f^{2}}|\operatorname{Re}[Y(f)]|,
$$

where $k_{B}$ is Boltzman's constant and $Y(f)$ is a complex admittance associated with $x(t)$. This complex admittance can be understood and computed as follows. Introduce a special set of generalized coordinates for the test-mass degrees of freedom-a set for which $x$ is one of the coordinates. (Since $x$ is not the coordinate of a normal mode of the test mass, these generalized coordinates will not be the usual ones associated with normal modes.) Apply to the test mass a generalized force $F(t)$ that drives the generalized momentum conjugate to $x$ but does not drive any of the other generalized momenta. This generalized force will show up as the following interaction term in the test-mass Hamiltonian:

$$
H_{\text {int }}=-F(t) x \text {. }
$$

This driving force, together with the test-mass internal elastic forces and internal dissipation, will generate a time evolution $x(t)$ of the observable $x$. Denote by $F(f)$ and $x(f)$ the Fourier transforms of the (arbitrary) driving force $F(t)$ and the observable's response $x(t)$. Then the admittance that appears in the thermal noise formula, Eq. (4), is

$$
Y(f)=2 \pi \imath f x(f) / F(f) .
$$

The physical nature of the driving force $F(t)$ can be deduced by inserting the definition (3) of the observable $x$ into the interaction Hamiltonian (5):

$$
H_{\mathrm{int}}=-\int P(\vec{r}) y(\vec{r}, t) d^{2} r
$$

where

$$
P(\vec{r}, t)=F(t) f(\vec{r}) .
$$

From Eq. (7) we see that the generalized force $F(t)$ consists of a pressure $P(\vec{r}, t)[\mathrm{Eq}$. (8)] applied to the test-mass surface. Note that the spatial distribution of this pressure is the same as LIGO's laser beam intensity profile.

The real part of the admittance, $\operatorname{Re}[Y(f)]$, describes the coupling of the test-mass dissipation to the observable $x$. We can see this most clearly by applying an oscillatory pressure $P(\vec{r}, t)=F_{0} \cos (2 \pi f t) f(\vec{r})$ to the test-mass face. From the response formula (6) we infer that the power $W_{\text {diss }}$ that this oscillatory pressure feeds into the test mass, and that the test mass then dissipates, is related to $|\operatorname{Re}[Y(f)]|$ by

$$
|\operatorname{Re}[Y(f)]|=\frac{2 W_{\text {diss }}}{F_{0}^{2}} .
$$

Substituting Eq. (9) into Eq. (4), we get

$$
S_{x}(f)=\frac{2 k_{B} T}{\pi^{2} f^{2}} \frac{W_{\mathrm{diss}}}{F_{0}^{2}} .
$$

Equation (10) is the most important equation of this paper. Let us reemphasize its physical content: (1) Apply an oscillatory pressure $P(\vec{r}, t)=F_{0} \cos (2 \pi f t) f(\vec{r})$ to the face of the test mass; (2) work out the average power $W_{\text {diss }}$ dissipated in the test mass under the action of this oscillatory pressure; (3) use $F_{0}$ and $W_{\text {diss }}$ in Eq. (10) to calculate $S_{x}(f)$.

This procedure is different from the one employed in previous calculations of internal thermal noise for the LIGO and VIRGO test masses [2,3,7]. The previous authors decomposed a test-mass motion into normal elastic modes; then they calculated the contribution of each mode to $S_{x}$ independently and added up these contributions. This method of "normal-mode decomposition"' works fine in many cases, but it has two drawbacks.

(1) The fundamental assumption in this method is that different normal modes have independent Langevin forces. This assumption is correct only if the sources of friction are homogeneously distributed over the test-mass volume. It breaks down if the defects are more concentrated in one 
place than in others - for example, when there is significant damping concentrated in the test-mass surface. We will return to this in Sec. IV.

(2) For a small laser beam diameter the sum over normal modes converges very slowly, and so one has to sum over many modes, which may be computationally expensive. By contrast, using the new method described in this paper, one can write down a simple analytic expression for the lowfrequency noise in the case of a narrow laser beam. In the next section we derive this expression and make comparison with the normal-mode decomposition results derived in [3].

\section{THERMAL NOISE DUE TO HOMOGENEOUSLY DISTRIBUTED DAMPING}

Consider the case where all the friction in the test mass comes from homogeneously distributed damping. It is conventional to characterize such friction by an imaginary part of the material's Young's modulus:

$$
E=E_{0}[1+\imath \phi(f)]
$$

$\phi(f)$ is called the material's "loss angle." It is suspected $[8,2]$ that for fused silica, which will be used in LIGO's test masses, $\phi$ might be independent of frequency within LIGO's detection band (but there is no evidence for such behavior of $\phi$ for high-quality resonators - see [9] for some healthy scepticism). In this $f$-independent case the damping is called "structural.",

To calculate the thermal noise for homogeneous dissipation, we express $W_{\text {diss }}$ in Eq. (10) as

$$
W_{\mathrm{diss}}=2 \pi f U_{\max } \phi(f),
$$

where $U_{\max }$ is the energy of elastic deformation at a moment when the test mass is maximally contracted or extended under the action of the oscillatory pressure of Eq. (8).

LIGO's detection frequencies $(10-300 \mathrm{~Hz})$ are much lower than the eigenfrequencies of the test-mass normal modes (the lowest of which is $\sim 6 \mathrm{kHz}$ ); so we can assume constant, nonoscillating pressure $P(\vec{r})=F_{0} f(\vec{r})$ when evaluating $U_{\max }$.

In the case when the beam profile is Gaussian and the center of the light spot coincides with the center of the transverse coordinates, we have

$$
f(\vec{r})=\frac{1}{\pi r_{0}^{2}} e^{-r^{2} / r_{0}^{2}},
$$

where $r_{0}$ is the radius of the laser beam. When the characteristic size of the test mass $R$ is much greater than $r_{0}$, we can approximate the test mass as an infinite half-space in order to find $U_{\max }$. The Appendix uses elasticity theory to derive $U_{\max }$ in this case [cf. Eq. (A5)]:

$$
U_{\max }=\frac{F_{0}^{2}}{\pi^{2} E_{0} r_{0}}\left(1-\sigma^{2}\right) I\left[1+O\left(\frac{r_{0}}{R}\right)\right],
$$

where $E_{0}$ and $\sigma$ are the Young's modulus and Poisson ratio of the material, respectively, and $I \simeq 1.87322$. Here $O\left(r_{0} / R\right)$ is a correction due to the finite size of the cylinder. Putting Eqs. (14) and (12) into Eq. (10), one gets

$$
S_{x}(f)=\frac{4 k_{B} T}{f} \frac{1-\sigma^{2}}{\pi^{3} E_{0} r_{0}} I \phi\left[1+O\left(\frac{r_{0}}{R}\right)\right] .
$$

Below we take the numerical values ${ }^{1}$ used by Gillespie and Raab [3]: $r_{0}=1.56 \mathrm{~cm}, E_{0}=7.18 \times 10^{10} \mathrm{~Pa}, \sigma=0.16$, $\phi=10^{-7}$, a mirror diameter of $25 \mathrm{~cm}$, and the mirror length of $10 \mathrm{~cm}$. Gillespie and Raab, after summing over the relevant $\sim 30$ modes, get

$$
S_{x}^{\mathrm{GR}}(100 \mathrm{~Hz}) \simeq 8.0 \times 10^{-40} \mathrm{~m}^{2} / \mathrm{Hz}
$$

Our analytical approximation (15) (which should be valid to within $\sim 10 \%$ in this case) gives

$$
S_{x}(100 \mathrm{~Hz}) \simeq 8.7 \times 10^{-40} \mathrm{~m}^{2} / \mathrm{Hz} .
$$

Notice that our analytic expression in Eq. (15) gets more exact when $r_{0} / R \rightarrow 0$, whereas, by contrast, the sum over modes converges more slowly and gets more complicated.

The ratio $r_{0} / R$ may turn out to be of order unity in real experiments. In this case, Eq. (15) can only be used for order-of-magnitude estimates. To work out the exact value of the internal thermal noise, one would need to calculate $U_{\max }$ numerically. We have done such a numerical computation using finite-element techniques. More specifically, we have used finite-element software called PDEASE2D (version 3.0), which runs as part of MASCYMA (Version 2.1), to solve the elasticity equations for the loaded mirror and to compute $U_{\max }$ and, by virtue of Eqs. (12) and (10), $S_{x}$. The exact answer for the mirror and light spot parameters given above is

$$
S_{x}(100 \mathrm{~Hz})=8.76 \times 10^{-40} \mathrm{~m}^{2} / \mathrm{Hz}
$$

which is consistent (better than expected) with our analytical approximation.

The purpose of the present section is to convince the reader that the method presented in this paper is correct and could be computationally cheaper than the normal-mode expansion. The next section concentrates on the cases where a direct application of the FD theorem can be crucial for getting the right results, and the method of normal-mode decomposition fails.

\section{CASE OF SURFACE DAMPING}

In this section we study thermal noise due to surface losses-caused, e.g., by inadequate polishing or by a lossy mirror coating.

From Eq. (10) we see that the key quantity in the thermal noise calculation is the power dissipated in the test mass when an oscillating pressure is applied to the laser beam spot on the test-mass surface. The power dissipated at each point of the material is proportional to the square of the stress at this point. Most of the surface stress is in or near the spot to which the pressure is applied, and so

\footnotetext{
${ }^{1}$ Note that our definition of the beam radius (location where intensity has fallen to $1 / e$ of its central value) differs by $\sqrt{2}$ from the beam radius of Ref. [3] (location of 1/e amplitude falloff).
} 


$$
W_{\text {diss }}^{\text {coating }} \propto\left(\frac{F_{0}}{r_{0}^{2}}\right)^{2} r_{0}^{2}=\frac{F_{0}^{2}}{r_{0}^{2}} \text {. }
$$

Thus the thermal noise due to the surface damping scales like

$$
S_{x}(\text { boundary }) \propto 1 / r_{0}^{2} .
$$

For comparison, the thermal noise due to bulk damping [Eq. (15)] scales as

$$
S_{x}(\text { bulk }) \propto 1 / r_{0} .
$$

Thus, as the spot size decreases, the thermal noise due to surface damping grows faster than that due to bulk damping.

Contrast this conclusion with the intuition one gets from normal-mode decomposition. There one is concerned with how much the surface contributes to the quality factors ( $Q$ 's) of the normal modes. For a typical mode the strain at the surface is at most of the same order as the characteristic strain inside the test mass (likely, much less for the first few modes, because of the free boundary condition). Therefore, one would presume that the surface contributes no more than some mode-independent fraction of the test mass's $Q$ 's. In order of magnitude this fraction should be the ratio of the power dissipated in the surface to that in the bulk if one applies an oscillating pressure uniformally to the whole surface, which in the context of our method corresponds to a beam radius of $R$. Therefore the normal-mode estimate of the surface thermal noise is at least $r_{0} / R$ less than the correct value.

Current experiments show that the mirror coating does not contribute significantly to the $Q$ 's of the test-mass normal modes. The conclusion commonly made is that coating is also not likely to contribute significantly to the internal thermal noise. The above analysis shows that this conclusion is not justified and that there might be a significant contribution of the coating to the internal thermal noise, despite the fact that $Q$ 's are not significantly changed.

\section{DISCUSSION AND CONCLUSION}

The normal-mode decomposition of the thermal noise is exact when the defects are distributed homogeneously through the volume of the test mass. However, as was shown explicitly in Sec. IV for the case of surface losses, when the defect distribution is not homogeneous, the normal-mode decomposition may be misleading, and a direct application of the fluctuation-dissipation theorem is required.

Thermal noise is ultimately linked to friction in the test mass; this friction is caused by various (structural and otherwise) defects. Those defects which are closer to the beam spot will contribute more to the thermal noise that is read out by the laser-beam phase shift. Although this fact is a direct consequence of the formalism developed in this paper, we would like to give an intuitive example in order to emphasize this point.

Consider, for the sake of simplicity, a one-dimensional elastic test mass with two identical defects $A$ and $B$, as shown on Fig. 1; $A$ is closer to the beam spot than $B$. Each of these defects creates a random stress which pushes apart or pulls together the left and right (relative to the defect) parts

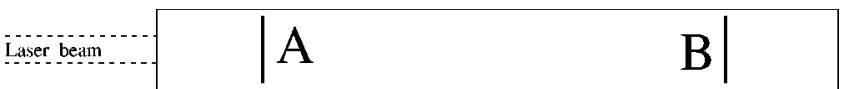

FIG. 1. Identical defects $A$ and $B$ create fluctuating stress in different parts of the test mass. The stress created by defect $A$ will influence the phase shift of the laser beam readout more than the stress created by defect $B$, although both $A$ and $B$ make identical contributions to the $Q$ 's of the test-mass elastic modes.

of the test mass. By conservation of momentum, the part of the test mass which is lighter will respond more to the random stress than the other part; therefore $\operatorname{defect} A$ will have a larger effect on the optical readout than $B$.

Note that if the defects $A$ and $B$ are positioned symmetrically with respect to the center of the test mass, they will have the same effect on the $Q$ 's of all elastic modes (we assume for simplicity that only one-dimensional longitudinal modes are present_-and all of them are either symmetric or antisymmetric with respect to the center). Therefore, the normal-mode decomposition applied to the test mass with just one defect- $A$ or $B-$ would give the same result for the thermal noise as read by the laser. Clearly, we have found yet another illustration of the breakdown of the normal-mode decomposition .

The considerations presented above lead to the following advice for real experiments: Keep the neighborhood of the laser beam spot as clean of defects as possible.

Not only does our direct application of the fluctuationdissipation theorem have broader validity than the normalmode decomposition; it is also computationally simpler. In the case of homogeneous structural damping it yields a simple analytical expression for the internal thermal noise spectrum [cf. Eq. (15)]:

$$
S_{x}(f)=\frac{4 k_{B} T}{f} \frac{1-\sigma^{2}}{\pi^{3} E_{0} r_{0}} I \phi\left[1-O\left(\frac{r_{0}}{R}\right)\right] .
$$

This result is consistent with the numerical sum over modes done in Ref. [3] and is accurate when the radius of the laser beam is small relative to the size of the test mass, i.e., in the regime when the sum over modes converges especially slowly. When $r_{0} / R$ is not small, a numerical solution of the elasticity equations to deduce the dissipation power $W_{\text {diss }}$, and thence the thermal noise (10), is straightforward and is probably also much simpler than performing a sum over modes.

\section{ACKNOWLEDGMENTS}

This work would not have been possible without discussions and help from Vladimir Braginsky, Ron Drever, Darrell Harrington, Nergis Mavalvala, Fred Raab, Glenn Soberman, and Kip Thorne. In particular, Glenn Soberman suggested the method of integration in Eq. (A4), and Kip Thorne carefully reviewed the manuscript and made a few significant corrections and suggestions. This work was supported in part by NSF Grant No. PHY-9424337. 


\section{APPENDIX: THE STRAIN ENERGY IN A TEST MASS SUBJECTED TO A GAUSSIAN DISTRIBUTED SURFACE PRESSURE}

The objective of this appendix is to derive Eq. (14) of Sec. III for the energy of elastic strain in a cylindrical test mass when the pressure $P(\vec{r})=F_{0} f(\vec{r})$ is applied to one of its circular faces. (As was discussed in Sec. III, we can assume that the pressure is constant in time since LIGO's detection frequencies are much lower than the lowest normalmode frequency.) For a circular laser beam with a Gaussian intensity profile $f(\vec{r})$ is given by [cf. Eq. (13)]

$$
f(\vec{r})=\frac{1}{\pi r_{0}^{2}} e^{-r^{2} / r_{0}^{2}}
$$

where we assume that the center of the light spot coincides with the center of the test-mass circular face.

If the radius of the laser beam $r_{0}$ is small compared to the size of the test mass, we can approximate the test mass by an infinite elastic half-space. Then our calculation of the elastic energy is correct up to a fractional accuracy of $O\left(r_{0} / R\right)$, where $R$ is the characteristic size of the test mass.

Let $y(\vec{r})$ be the normal displacement of the surface at location $\vec{r}$ under the action of the pressure $P(\vec{r})$. In the linear approximation of small strains,

$$
y(\vec{r})=\int G\left(\vec{r}, \overrightarrow{r^{\prime}}\right) P\left(\overrightarrow{r^{\prime}}\right) d^{2} r^{\prime},
$$

where $G\left(\vec{r}, \overrightarrow{r^{\prime}}\right)$ is a Green's function. The calculation of $G$ is a nontrivial albeit standard exercise in elasticity theory [10], which gives

$$
G\left(\vec{r}, \overrightarrow{r^{\prime}}\right)=\frac{1-\sigma^{2}}{\pi E_{0}} \frac{1}{\left|\vec{r}-\overrightarrow{r^{\prime}}\right|}
$$

where $\sigma$ is the Poisson ratio and $E_{0}$ the Young's modulus of the material. The elastic energy stored in the material is

$$
\begin{aligned}
U_{\max } & =\frac{1}{2} \int P(\vec{r}) y(\vec{r}) d^{2} r=\frac{1}{2} \frac{1-\sigma^{2}}{\pi E_{0}} \int \frac{P(\vec{r}) P\left(\overrightarrow{r^{\prime}}\right)}{\left|\vec{r}-\vec{r}^{\prime}\right|} d^{2} r d^{2} r^{\prime} \\
& =\frac{1}{2} \frac{1-\sigma^{2}}{\pi^{3} E_{0} r_{0}^{4}} F_{0}^{2} \int \frac{e^{-\left(r^{2}+r^{\prime 2}\right) / r_{0}^{2}}}{\sqrt{r^{2}+r^{\prime 2}-2 r r^{\prime} \cos \theta}} d^{2} r d^{2} r^{\prime}, \quad(\mathrm{A} 4)
\end{aligned}
$$

where $\theta$ is the angle between $\vec{r}$ and $\overrightarrow{r^{\prime}}$. The integral in the last term of Eq. (A4) (as was pointed out by Glenn Sobermann) can be taken by introducing "polar' coordinates $R$ and $\phi: r=R \cos \phi, r^{\prime}=R \sin \phi$. One then integrates out the radial part of the integrand and expands the remaining angular part in a power series with respect to $\cos \theta$; termwise integration of this power series finally yields Eq. (14) [up to a fractional error of $\left.O\left(r_{0} / R\right)\right]$

$$
U_{\max } \simeq \frac{F_{0}^{2}}{\pi^{2} E_{0} r_{0}}\left(1-\sigma^{2}\right) I,
$$

where

$$
I=\frac{\pi^{3 / 2}}{4}\left[1+\sum_{n=1}^{\infty} \frac{(4 n-1) ! !}{(2 n) ! 4^{n}(2 n+1)}\right] \simeq 1.87322 .
$$

It can be shown that if, instead of an infinite half-space, we consider a finite cylindrical test mass, the leading fractional correction to the elastic energy is of the order $O\left(r_{0} / R\right)$.
[1] A. Abramovici et al., Science 256, 325 (1992); C. Baradaschia et al., Nucl. Instrum. Methods Phys. Res. A 289, 518 (1990).

[2] P. R. Saulson, Phys. Rev. D 42, 2437 (1990).

[3] A. Gillespie and F. Raab, Phys. Rev. D 52, 577 (1995).

[4] G. I. Gonzalez and P. R. Saulson, J. Acoust. Soc. Am. 96, 207 (1994).

[5] H. B. Callen and T. A. Welton, Phys. Rev. 83, 34 (1951).
[6] N. Nakagawa et al., Rev. Sci. Instrum. 68, 1 (1997).

[7] F. Bondu and J. Y. Vinet, Phys. Lett. A 198, 74 (1995).

[8] A. Gillespie and F. Raab, Phys. Lett. A 178, 357 (1993).

[9] V. B. Braginsky et al., Phys. Lett. A 218, 164 (1996), and references therein; (private communication).

[10] Equation (8.19) of L. D. Landau and E. M. Lifshitz, Theory of Elasticity (Pergamon, New York, 1986). 